\title{
La Simulación como estrategia didáctica en las prácticas de formación docente. Experiencia en la carrera Ciencias Sociales.
}

\author{
Dr. Julio César Orozco Alvarado \\ Doctor en Educación e Interveción Social \\ UNAN-Managua \\ jorozcoa@hotmail.com
}

\section{Lic. Adilia Aracelly Cruz Acevedo}

Ciencias de la Educación con mención en Ciencias Sociales

UNAN-Managua

adiliacruz2@hotmail.com

\section{Lic. Adolfo Alejandro Díaz Pérez}

Ciencias de la Educación con mención en Ciencias Sociales

UNAN-Managua

adolfoalejandro73@yahoo.com

Fecha de recepción: 28 de febrero, 2020 / Fecha de aceptación: 20 de mayo, 2020

https://doi.org/10.5377/torreon.v9i25.9851

Palabras clave: Ciencias Sociales, competencias docentes, estrategias didácticas, simulación.

\section{RESUMEN}

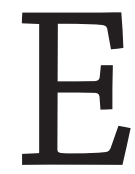

1 presente artículo ${ }^{1}$ de innovación educativa surge de un proceso de reflexión pedagógica que tuvo como objetivo utilizar la simulación como estrategia didáctica para desarrollar competencias pedagógicas en docentes en formación del área de ciencias sociales en la asignatura Prácticas de Especialización de la carrera Ciencias Sociales. La metodología consistió en desarrollar un estudio mixto, con énfasis en lo cualitativo,

1. Artículo de investigación producto de una sistematización de una experiencia didáctica en la asignatura Prácticas 
aplicando técnicas de investigación como observación, encuesta y entrevistas a la unidad de análisis de la investigación que estuvo compuesta por veintiocho estudiantes matriculados en la asignatura. La intervención didáctica dio como resultados el desarrollo de las diez competencias docentes planteadas en la asignatura, lo cual se evidenció en las simulaciones que los estudiantes realizaron durante el curso de la asignatura. Este estudio demuestra que la simulación es una estrategia didáctica que favorece la formación de profesionales de la educación y que permite que los protagonistas asuman roles semejantes a los que deben asumir en la realidad y por ende, apropiarse del rol, conocimientos, actitudes, valores y habilidades que el medio requiere para desempeñarse efectivamente.

\section{INTRODUCCIÓN}

La presente investigación es producto de una experiencia didáctica realizada en la asignatura Práctica de Especialización de la carrera Ciencias Sociales, Facultad de Educación e Idiomas de la Universidad Nacional Autónoma de Nicaragua, Managua (UNAN-Managua). La investigación surge de la necesidad de desarrollar competencias docentes en el profesorado en formación a través de la asignatura Prácticas de Especialización, la cual contempla, además del trabajo tutorial por parte del docente en el aula de clases, ir a las aulas de clase de Educación secundaria a poner en práctica los conocimientos científicos y metodológicos didácticos adquiridos durante los cinco semestres anteriores cursados en la carrera.

En este sentido, es preciso señalar que la asignatura en mención correspondía desarrollarse durante el II semestre 2018 (de agosto a noviembre), sin embargo, debido a las condiciones sociopolíticas que atravesó el país, este se desarrolló entre los meses de enero a abril 2019, asimismo, como consecuencia de este contexto sociopolítico únicamente se hacía posible desarrollar la parte teórica en el aula de clase y no la parte práctica de intervención educativa en las aulas de clases de Educación secundaria, por lo tanto, el equipo de investigadores consensuó desarrollar las prácticas pedagógicas en el aula de clase mediante simulaciones docentes, es decir, que los estudiantes (profesores en formación), pusieran en prácticas sus conocimientos disciplinares y didácticos impartiendo docencia a su mismo grupo de clase.

A partir de este nuevo enfoque asumido en el proceso de aprendizaje, se realizó una revisión bibliográfica sobre la implementación de la simulación en los procesos de formación de profesionales de la educación, y se encontraron diversos estudios aplicados en distintas áreas del conocimiento, por citar uno, la experiencia desarrollada por López, Ramos, Pato y López Álvarez (2012) en el área de la medicina, quienes desarrollaron La simulación clínica como herramienta de aprendizaje, la cual permite que los estudiantes desarrollen competencias profesionales sin necesidad de estar en el propio medio ocupacional, al respecto el estudio concluye que: "hasta ahora era con la experiencia clínica con la que se llegaban a adquirir estas habilidades, pero tras 
la implantación de la cultura de la seguridad este tipo de aprendizaje ya no es admisible. Es por tanto la simulación la herramienta eficaz mediante la cual es posible acortar dichas curvas. La enseñanza en entornos de este tipo ha de estar integrada perfectamente en el currículum con la enseñanza teórica y práctica y en consonancia con los objetivos del aprendizaje”.

Sin embargo, además de obtener resultados significativos en el área de la medicina, en el campo de la educación también se encontraron diversos estudios en donde se utiliza la simulación como estrategia didáctica para la formación de profesionales en distintas especializaciones, ejemplo de ello es el estudio de Sánchez (2013) titulado La simulación como método de enseñanza y aprendizaje, donde se encontraron los siguientes hallazgos: (1) La selección de la simulación como estrategia didáctica contribuyó para que los estudiantes desarrollaran la confianza y seguridad necesaria para pararse frente a un grupo y «actuar como profesor» relacionando los aspectos teóricos ya adquiridos con la práctica en clase; (2) Posibilitó posicionarse, tomar decisiones, asumir roles y comprender situaciones de la dinámica institucional y áulica, y (3) Permitió que los docentes se adentraran en el sentir, en el hacer y en el pensar de los alumnos y además, recuperar de la práctica de simulación un espacio de análisis y reflexión conjunta.

Asimismo, en el área de la didáctica de las ciencias sociales en el contexto educativo nicaragüense, estudios como el de Orozco y Díaz (2017) ya expresaban por sí el valor de la simulación como estrategia didáctica en los procesos de aprendizajes, dicha investigación fue una innovación educativa que se llevó a cabo en el Colegio Público Esquipulas de Managua con estudiantes de Educación secundaria quienes asumiendo el rol de personajes históricos generaron procesos de empatía y lograron aprender significativamente el contenido de aprendizaje, lo cual indica que la simulación es una estrategia de aprendizaje que propicia el desarrollo de competencias y para generar procesos empáticos con la realidad, sin necesidad de estar inmerso en la misma.

No obstante, con base en estos hallazgos, el equipo de investigadores se planteó como objetivo desarrollar competencias docentes en la asignatura Prácticas de Especialización mediante la implementación de la simulación como estrategia didáctica, es decir, el proceso de investigación partió de la hipótesis que la simulación como estrategia didáctica permite desarrollar competencias docentes en la formación del profesorado de ciencias sociales, para ello la asignatura se planificó con seis encuentros teóricos relacionados con los aspectos básicos para ejercer la profesión docente en donde se debían desarrollar contenidos vinculados con el rol del docente en los procesos de aprendizaje, las fases del proceso de aprendizaje, el decálogo del docente eficaz entre otros; y los restantes nueve encuentros del semestre se dedicaron a realizar simulaciones de las diferentes asignaturas de Ciencias Sociales que se imparten en Educación secundaria. 
Por consiguiente, en el presente artículo se sistematiza esta experiencia didáctica llevada a cabo con estudiantes de tercer año de la carrera Ciencias Sociales de la Facultad de Educación e Idiomas de la Universidad Nacional Autónoma de Nicaragua, Managua (UNAN-Managua), y para ello se ha diseñado un referente metodológico y teórico con el cual se rige la investigación.

\section{METODOLOGÍA}

\section{Enfoque y alcance de la investigación}

Siguiendo los referentes teóricos de Hernández, Fernández y Baptista (2014), la investigación se sitúa en un enfoque mixto precisamente porque consiste en un conjunto de procesos sistemáticos, empíricos y críticos de investigación que implican la recolección y el análisis de datos cuantitativos y cualitativos.

En el presente caso, la investigación se considera mixta porque procesa datos de origen cuantitativo y cualitativo. Con relación a lo primero, se aplicó un cuestionario para determinar las competencias que el estudiantado había desarrollado durante el transcurso de la asignatura, y en referencia a la perspectiva cualitativa se realizó una entrevista con 10 estudiantes que cursaron la asignatura, esto con el propósito de profundizar en el análisis de la incidencia de la simulación como estrategia didáctica para la formación del profesorado de ciencias sociales, y se realizaron bitácoras producto de la observación docentes para dar seguimiento a todo el proceso de aprendizaje que se estaba gestando, asimismo, se utilizaron listas de cotejo para evidenciar las competencias desarrolladas por el estudiantado.

\section{Unidades de análisis}

La unidad de análisis de la presente investigación está constituida por los 28 estudiantes matriculados en la asignatura Prácticas de Especialización de la Carrera Ciencias Sociales, estos, siguiendo los planteamientos de Bernal (2010), son «la parte de la población que se selecciona, de la cual realmente se obtiene la información para el desarrollo del estudio y sobre la cual se efectuarán la medición y la observación de las variables objeto de estudio» (p.161).

Los criterios de selección fue que los estudiantes hubiesen aprobada la asignatura Didáctica de las Ciencias Sociales, la cual se cursa en el semestre anterior, y que estuviesen matriculado en la asignatura. Esto garantizó que el estudiantado al momento de la intervención didáctica dominara los conocimientos básicos sobre la enseñanza de las ciencias sociales, y que potenciaran tales conocimientos en el desarrollo teórico de la asignatura, y finalmente, que los aplicaran al realizar la simulación en el aula de clase. 


\section{Técnicas de recolección}

En el proceso de investigación se aplicaron tres técnicas de investigación: observación, encuesta y entrevistas. En el caso de la primera, apegados a Arias (2012), "es una técnica que consiste en visualizar o captar mediante la vista, en forma sistemática, cualquier hecho, fenómeno o situación que se produzca en la naturaleza o en la sociedad, en función de unos objetivos de investigación preestablecidos» (p.69). Esta técnica mediante el instrumento lista de cotejo y bitácora, permitió registrar las competencias que el estudiantado desarrolló durante el proceso de aprendizaje de las Prácticas de Especialización.

Respecto a la encuesta, siguiendo los aportes generados en Arias (2012), se concibe como un instrumento o formato en papel con una serie de preguntas que debe ser llenado por el encuestado sin intervención del encuestador. Este cuestionario se aplicó a 28 estudiantes que cursaron la asignatura con el objetivo de indagar las competencias docentes desarrolladas durante la asignatura, y en cuanto a la entrevista, Arias (2012) también expresa que es «una técnica basada en un diálogo o conversación 'cara a cara', entre el entrevistador y el entrevistado acerca de un tema previamente determinado, de tal manera que el entrevistador pueda obtener la información requerida» (p.73). Esta técnica se aplicó a 10 estudiantes que cursaban la asignatura, y permitió profundizar en aspectos relacionados con el impacto que tuvo la implementación de la simulación en el desarrollo de competencias docentes.

\section{RESULTADOS}

\section{Asignatura Prácticas de Especialización}

Las Prácticas de Especialización es una asignatura contemplada en el VI semestre del Plan de Estudios de la Carrera Ciencias Sociales de la Universidad Nacional Autónoma de Nicaragua, Managua (UNAN-Managua), y de acuerdo al Programa de la asignatura de Prácticas de Especialización (2014), estas deben ser vista como una oportunidad para la puesta en práctica de los conocimientos disciplinares del área de Geografía, Historia, Economía, Filosofía y Sociología; así como los conocimientos psicopedagógicos adquiridos en las asignaturas cursadas en los semestres anteriores lo que les permitirá un desempeño efectivo y eficiente como docente, asimismo, las Prácticas de Especialización permiten interactuar con el estudiantado de secundaria por medio del ejercicio de la docencia.

También es importante destacar que el objetivo principal de esta asignatura es que los docentes en formación tengan contacto directo con las instituciones de Educación secundaria y puedan impartir docencia en el área de Ciencias Sociales y de esta manera tengan la oportunidad de conocer el manejo de los documentos curriculares que orienta el Ministerio de Educación para el proceso de enseñanza-aprendizaje en las aulas de clases. En definitiva, de acuerdo con 
el programa de asignatura de Prácticas de Especialización (2014), este pretende que el profesor en formación logre desarrollar las siguientes competencias: (1) dominio científico de los contenidos de las asignaturas de ciencias sociales, (2) vínculo de los contenidos de aprendizajes con el contexto social, (3) control del grupo de clase, (4) elaboración de materiales didácticos, (5) utilización de recursos didácticos en el proceso de aprendizaje, (6) aplicación de instrumentos de evaluación de los aprendizajes, (7) planificación de clases con enfoque por competencia, (8) implementación de estrategias didácticas innovadoras, (9) aplicación de distintos tipos y formas de evaluación de los aprendizajes y (10) modelaje de metodologías participativas en los procesos de aprendizajes.

En este sentido, la asignatura se ha diseñado en dos momentos; uno teórico que consiste en el abordaje de contenidos relacionados con la ética profesional del docente y contenidos relacionados al dominio didáctico, y otro momento que consiste en visita a las aulas de clases de Educación secundaria para impartir docencia en el área de ciencias sociales (Programa de la asignatura de Prácticas de Especialización, 2014), sin embargo, como se explicó en los apartados anteriores, se utilizó la simulación como estrategia didáctica para que el estudiantado alcanzara los objetivos prácticos planteados en el programa de asignatura sin necesidad de ir a los centros educativos de Educación secundaria.

\section{La simulación como estrategia de aprendizaje}

La simulación es una estrategia de aprendizaje grupal que permite que los estudiantes desarrollen procesos empáticos y se empoderen de roles en la representación de circunstancias, hechos o acontecimientos. Esta estrategia ha sido utilizada en diversas áreas del conocimiento con el fin de generar experiencias significativas en el estudiantado para que este conocimiento sea interiorizado, duradero y aplicado a otras situaciones. Al respecto, diversos autores han teorizado sobre esta estrategia didáctica brindado sus aportes y aprendizajes adquiridos por medio de la implementación de esta estrategia en su práctica docente. A continuación se presentan algunas de ellas:

En Pimienta (2012), se concibe la simulación como «una estrategia que pretende representar situaciones de la vida en las que participan los alumnos actuando roles con la finalidad de dar solución a un problema o, simplemente, para experimentar una situación determinada» (p.130); de manera semejante, Davini (2008) sostiene que la simulación es un «método de enseñanza que se propone acercar a los alumnos a situaciones y elementos similares a la realidad, pero en forma artificial, a fin de entrenarlos en habilidades prácticas y operativas cuando las encaran en el mundo real» (p. 144).

Ambas concepciones comparten referentes comunes porque la simulación es una estrategia didáctica que tiene el propósito de preparar al estudiantado para que afronten 
situaciones reales sin necesidad que estos vayan al medio en sí, esto es ventajoso porque propicia el desarrollo de competencias y consolidación de las nociones que tiene el estudiantado sobre la realidad en cualquier área del conocimiento. En el caso de la presente investigación se persigue como fin que los estudiantes consoliden sus conocimientos y competencias docentes y que puedan experimentar lo que se vive en el aula de clase sin necesidad de estar propiamente con el estudiantado en un aula de clase, es decir, la simulación funge como una especie de laboratorio en el que se experimentan los fenómenos que se dan en los procesos de aprendizajes de Educación secundaria.

\section{El valor didáctico de la simulación}

En las diversas literaturas consultadas sobre la utilización de la simulación como estrategia de aprendizaje se puede observar que esta estrategia es utilizada en las distintas áreas de educación y formación docente como Ciencias Sociales, Matemáticas, Lengua y Literatura, Física, Ciencias Naturales etc., pero también en otras ciencias como la Medicina, Odontología, Derecho, Arqueología etc. De esta amplia experiencia de distintos investigadores se han retomado diversas conclusiones, aportes y hallazgos para determinar los distintos fines que tiene la simulación como estrategia de aprendizaje en los procesos de formación de profesionales de las distintas áreas del conocimiento científico:

- La simulación favorece las prácticas innovadoras, resolución de problemas, y facilita la transferencia de conocimientos, habilidades y capacidades a diversas áreas de conocimiento (Pimienta, 2012).

- La simulación es una estrategia que supone tomar decisiones sobre diferentes dimensiones de la realidad (Sánchez, 2013).

- La simulación permite al estudiante desarrollar un aprendizaje autónomo, significativo, vicario, cooperativo, reflexivo y habilidades de pensamiento crítico. (Urra, Sandoval e Irribarren, 2017).

La perspectiva planteada por estos autores (Pimienta, 2012; Sánchez, 2013; Urra, Sandoval e Irribarren, 2017) apuntan hacia elementos comunes: la simulación es una estrategia propicia para el desarrollo de las distintas competencias específicas de cada área del conocimiento y facilita la formación de profesionales con los conocimientos, habilidades y destrezas necesarias para afrontar los fenómenos presentes en la realidad, en este sentido, es una estrategia que vincula significativamente los procesos de aprendizajes adquiridos en el aula de clase de manera teórica con los problemas reales presentes en el contexto de cada profesión, adquiriendo así una dimensión teórica-práctica la formación de los profesionales. 


\section{Caracterización del estudiantado}

Los estudiantes matriculados para cursar la asignatura Prácticas de Especialización fueron veintiocho, los cuales proceden de diversos municipios de los departamentos de Managua y Masaya. De este total de estudiantes, cabe mencionar que el 57\% está laborando en el sistema de educación nicaragüense, distribuidos en centros públicos y privados, y el $43 \%$ no laboran en educación. También es importante agregar que los estudiantes que laboran en educación están insertos en distintas modalidades educativas, por ejemplo, el diagnóstico realizado proporcionó la siguiente información: el 7\% imparte docencia en preescolar, el 14\% en educación primaria, el $29 \%$ en educación secundaria y el $7 \%$ en secundaria a distancia.

En este sentido, es importante destacar que un poco más de la mitad de los estudiantes inscritos en la Prácticas de Especialización tenían experiencia docente, por tal razón, han desarrollado distintas competencias docentes para llevar a cabo los procesos de aprendizajes, sin embargo, también se contó con un $43 \%$ de estudiantes que no tenían experiencia docente y que solamente habían acumulado experiencia a través de la asignatura Prácticas de Familiarización, la cual es una asignatura precedente y requisito para cursar las Prácticas de Especialización, ya que en esta asignatura los estudiantes van a los centros educativos a observar clases de ciencias sociales y entrevistar a los docentes, asimismo, los estudiantes asisten como oyentes a los Encuentros Pedagógicos de Interaprendizaje (EPI) para conocer cómo se desarrolla el proceso de planificación educativa mensual.

\section{DISCUSIÓN}

\section{Estrategias didácticas aplicadas en la simulación}

Las estrategias didácticas que los estudiantes aplicaron en sus respectivas simulaciones fueron diversas, para ello, primeramente realizaron una revisión documental para luego hacer una propuesta innovadora para la enseñanza de las ciencias sociales, con base en esto, el estudiantado implementó las siguiente estrategias didácticas: Lecturas comentadas, exposiciones, resolución de guía de estudios, estudio de casos, elaboración de collage, elaboración de álbum, trabajo en equipo, dictado de contenidos, resolución de clases prácticas, elaboración de maquetas, simulación de hechos o sucesos históricos, interpretación de canciones, elaboración de esquemas (mapas conceptuales, redes temáticas, cuadro sinóptico, etc.), observación y análisis de vídeo; entre otras estrategias que les permitió llevar a la prácticas sus propuestas innovadoras para la mejora de los procesos de aprendizajes en las asignaturas de ciencias sociales. A continuación se describirá a manera de síntesis, por razones de extensión, las estrategias didácticas que más utilizaron los estudiantes en sus respectivas simulaciones: 


\section{Guías de estudios}

Las guías de estudios implementada por los estudiantes en la simulación parte de una propuesta innovadora realizada por Orozco y Díaz (2018), que es concebida como un «recurso didáctico compuesto por un conjunto de actividades de aprendizajes mediante las cuales el profesorado crea las condiciones didácticas para que el estudiantado se aproxime de manera autónoma al material de estudio o contenido objeto de aprendizaje» (p.59). En este sentido, la guía de estudio que elaboraron los estudiantes, tanto en su estructura interna como externa, fue innovadora porque no redactaron un conjunto de preguntas mecánicas, sino que diseñaron actividades de aprendizaje como: análisis de casos, análisis de epígrafes y análisis de imágenes; además, una vez resueltas las guías de estudios los estudiantes pasaban a una fase de socialización del conocimiento mediante conversatorios, plenarios o debates, esto con el fin de generar aprendizajes significativos en el estudiantado.

Con relación a esta estrategia didáctica, uno de los estudiantes que aplicó esta estrategia expresó lo siguiente: «La implementación de esta estrategia permitió la participación activa, facilitando así que los estudiantes sean los protagonistas en la construcción de su aprendizaje, de igual manera permitió la interacción alumno-contenido, alumno-alumno y alumno-maestro al momento de socializar las actividades planificadas».

\section{Simulación de hechos o sucesos históricos}

Los estudiantes también utilizaron la simulación como estrategia didáctica, en esta los estudiantes simularon las funciones de Faraón dentro de la civilización egipcia, lo cual permitió que protagonizaran este hecho interactuando con el grupo de clase y asumieran actitudes empáticas con este hecho histórico. Y esta idea coincide con la perspectiva de Ibáñez (2015), quien sostiene que mediante la simulación se genera empatía y se desprenden los prejuicios prepotentes del presente hacia situaciones del pasado, intentando comprender la realidad de los hombres de otras épocas al igual que las problemáticas actuales.

\section{Interpretación de canciones}

Siguiendo los resultados de experiencias didácticas realizada por Díaz, Mendoza y Arce (2015), se concibe la música como un elemento didáctico que puede aplicarse en los espacios educativos para capturar la atención de los estudiantes y generar reflexión de contenidos. Por lo que, dentro de las simulaciones efectuadas, los estudiantes utilizaron la estrategia de interpretación de canciones, la cual se observó que generó interés en el aula de clase y permitió socializar el conocimiento y contextualizar el contenido de aprendizaje. Respecto a esta estrategia, uno de los estudiantes expresó lo siguiente: «Me gustó esta estrategia porque se realizaron dos cosas, primero analizar la canción, y luego vincularla con el contenido. Es decir, es muy buena técnica porque nos lleva a reflexionar no leyendo, sino escuchando». 


\section{Competencias docentes desarrolladas mediante la simulación}

Para determinar el grado de alcance de las competencias antes mencionadas se diseñó una lista de cotejo con una escala de puntuación la cual se utilizó para evaluar las simulaciones realizadas por el estudiantado. A continuación se presenta una escueta descripción del desarrollo de las competencias, esto por razones de extensión, y el total porcentual que alcanzó el estudiantado en cada una de ellas:

- Dominio científico de los contenidos de las asignaturas de ciencias sociales: Esta competencia se evidenció mediante la explicación y retroalimentación que realizaba el docente tutor acerca de los contenidos de aprendizajes. De acuerdo a los resultados de la lista de cotejo, el 95\% de los estudiantes mostraron dominio del contenido de aprendizaje.

- Vínculo de los contenidos de aprendizajes con el contexto social: Se observó que los docentes lograban contextualizar los contenidos de aprendizajes apoyándose en noticias coyunturales, videos y testimonios de personas sobre la coyuntura actual. En este sentido, de acuerdo a la lista de cotejo, el $87 \%$ de los estudiantes que participaron del estudio lograron vincular los contenidos con el contexto.

- Control del grupo de clase: Se presentaron actos de indisciplina que permitieron que el docente actuara con tolerancia y asertividad al crear un ambiente de aula propicio para el aprendizaje. Los resultados obtenidos indican que el 100\% de los estudiantes tuvieron dominio del grupo.

- Elaboración de materiales didácticos: Se evidenció que los docentes implementaron guías de estudios, fichas, dinámicas grupales y otros materiales didácticos realizados por ellos, a fin de generar un espacio de aprendizaje participativo. Al respecto uno de los participantes destacó la importancia de diseñar materiales didácticos: «logré desarrollar la preparación de mi plan de clases y desarrollar habilidades entre las cuales logré identificar la necesidad de practicar el orden, asumir compromiso al momento de preparar los materiales a utilizarse, así como la innovación que me permitió desarrollar mi contenido de la forma más dinámica e innovadora». De acuerdo a la lista de cotejo, el $82 \%$ de los estudiantes (docentes en formación) elaboraron materiales didácticos para el desarrollo de sus clases.

- Utilización de recursos didácticos en el proceso de aprendizaje: Los estudiantes de la práctica de especialización utilizaron mapas, esferas, líneas de tiempo, imágenes, diarios y objetos asociados a los contenidos de aprendizajes. Al respecto, uno de los estudiantes hizo énfasis en el valor didáctico de utilizar este tipo de materiales en los procesos de aprendizajes: «con las observaciones a cada grupo me di cuenta cómo se impartía una clase con diversas estrategias y cómo se aprovechaba cada una de ellas al hacer uso 
de material didáctico previamente elaborado, logrando así alcanzar el indicador de la clase». Según la escala de la lista de cotejo, el 86\% de los estudiantes utilizaron recursos didácticos en los procesos de aprendizajes

- Aplicación de instrumentos de evaluación de los aprendizajes: Entre los instrumentos de evaluación aplicados podemos destacar los siguientes: SQA (Lo que sé, lo que quiero aprender y lo que aprendí), KPSI (siglas del inglés, significa inventario de conocimientos previos de los estudiantes), RA-P-RP significa (RA Respuesta Anterior, P pregunta y RP Respuesta Posterior), lista de cotejo y rúbricas. Estos instrumentos de evaluación son innovadores en la enseñanza de las ciencias sociales, y permitió que los practicantes evaluaran con mayor precisión el desempeño de los estudiantes. En este sentido, el $76 \%$ de los estudiantes aplicaron estos instrumentos de evaluación en los procesos de aprendizajes.

- Planificación de clases con enfoque por competencia: Se realizó una revisión a los planes de clases de los docentes, en esto se evidenció la aplicación de un enfoque por competencias y la coherencia metodológica y didáctica de las actividades planificadas. Con respecto a esto, el 100\% de los practicantes implementó en su planificación didáctica el enfoque por competencias.

- Implementación de estrategias didácticas innovadoras: Se logró observar que las estrategias didácticas implementadas en la simulación fueron innovadoras, por citar el ejemplo de las guías de estudios descrito en el apartado anterior. Asimismo, en este aspecto los docentes en formación obtuvieron un 95\% de alcance en esta competencia.

- Implementación de distintos tipos y formas de evaluación de los aprendizaje: una propuesta innovadora fue implementar con más frecuencia la evaluación formativa y la coevaluación, lo cual es poco usual en el aprendizaje de las ciencias sociales, esto se evidenció en la simulación del contenido revolución industrial en donde los estudiantes participaron elaborando una línea de tiempo y un álbum en equipo sobre los principales inventos dados en esta época, luego presentaron en clase los resultados obtenidos donde un equipo evaluaría al otro mediante una rúbrica elaborada. En este aspecto, los estudiantes alcanzaron un $86 \%$ del desarrollo de esta competencia.

- Implementación de metodologías participativas en los procesos de aprendizajes: En las simulaciones realizadas, se pudo observar que los practicantes implementaron metodologías participativas acompañadas de dinámicas grupales motivacionales, entre las estrategias didácticas más utilizadas se pueden mencionar las siguientes: trabajo en equipo, debates, conversatorios, mesa redonda, simulación de hechos históricos, 
lecturas comentadas, plenarios. En este aspecto, los estudiantes alcanzaron un $70 \%$ del alcance de la competencia.

\section{CONCLUSIONES}

El presente estudio es resultado de un contexto social complejo que impuso distintas limitantes a los procesos de aprendizajes, sin embargo, siendo el docente un investigador e innovador de su práctica pedagógica, se deben trazar distintas alternativas que conlleven a alcanzar los fines educativos de cada asignatura, y esto fue precisamente lo que al equipo de investigadores los conllevó a replantear la metodología de la asignatura y llegar al hallazgo que la simulación es una estrategia didáctica que propicia la formación del profesorado. A continuación se detallan las conclusiones de este estudio:

- La simulación es una estrategia didáctica que favorece la formación de profesionales de la educación en diversas áreas del conocimiento. Mediante esta estrategia se llevan a cabo procesos empáticos que permite que los protagonistas asuman roles semejantes a los que deben asumir en la realidad, y por ende, apropiarse del rol, conocimientos, actitudes y habilidades que el medio requiere para desempeñarse efectivamente.

- Los estudiantes (docentes en formación) al realizar las simulaciones pudieron observar la efectividad de las diversas estrategias didácticas recopiladas mediante la revisión documental, mismas que fueron implementadas efectivamente por todo el grupo de clase. Esto expresa por sí mismo una nueva concepción de las prácticas docentes las cuales apuntan a desarrollar experiencias de aprendizajes novedosas en el aula de clase, haciendo frente así a las metodologías tradicionales con las cuales cotidianamente se llevan a cabo los procesos de aprendizajes de ciencias sociales en Educación secundaria.

- La función del docente tutor es indispensable en el proceso de formación de profesorado de ciencias sociales. En este caso, el rol del equipo de docentes investigadores consistió en brindar retroalimentación constante a los estudiantes, esto con el objetivo de posicionarlos frente al desarrollo de las diez competencias planteadas en el desarrollo de la asignatura.

- El docente es un investigador de su práctica pedagógica y las limitantes externas no son más que motivos para emprender una nueva innovación pedagógica que conlleve a alcanzar los fines previamente propuestos. A sabiendas de esto, es indispensable que nuestras prácticas docentes vayan acompañadas de nuevas incorporaciones metodológicas que conlleven a los mismos fines o bien, que permitan mejorar la calidad de los aprendizajes. 


\section{REFERENCIAS}

Arias, F. (2012). El proyecto de Investigación. Introducción a la metodología científica. (6ta ed.). Caracas: Episteme.

Bernal, C. (2010). Metodología de la investigación. (3ra ed.). Colombia: Pearson Educación.

Davini, M. (2008). Métodos de enseñanza: didáctica general para maestros $y$ profesores. Buenos Aires: Santillana.

Díaz, A., Mendoza, M., y Arce, L. (2015). Intervención Didáctica con estrategias de aprendizaje Innovadoras para generar Comprensión en la Disciplina Historia. Tesis para optar al grado de Licenciatura. Managua: UNAN-Managua.

Hernández, R., Fernández, C. y Baptista, M. (2014). Metodología de la Investigación. (6ta edición). México: McGRAW-HILL.

Ibáñez, J. (2015). La simulación en el aula: Juego y aprendizaje de la realidad. Tesis para optar al grado de Máster. España: Universidad de la Rioja.

López Sánchez, M., Ramos López, L., Pato López, O. y López Álvarez, S. (201). La simulación clínica como herramienta de aprendizaje. CIR MAY AMB, 18(1):25-29. http://www.asecma.org/ Documentos/Articulos/05_18_1_FC_ Lo\%C2\%A6\%C3\%BCpez.pdf

Orozco, J. y Díaz, A. (2017, marzo). La simulación como estrategia didáctica para desarrollar comprensión en la asignatura Historia. Intervención didáctica realizada en Educación secundaria. Revista Científica de FAREM-Estelí. Medio ambiente, tecnología y desarrollo humano, (6):4-13.

Orozco, J. y Díaz, A. (2018, junio). Un reto en la Innovación Pedagógica: Las guías de aprendizaje. Revista electrónica de Conocimientos, Saberes y Prácticas, 1(1):54-71. http://recsp.org/index.php/ recsp/article/view/4/5

Pimienta, J. (2012). Estrategias de enseñanza-aprendizaje. Docencia universitaria basada en competencias. México: Pearson.

Sánchez, M. (2013). La simulación como estrategia didáctica: Aportes y reflexiones de una experiencia en el nivel superior. Argentina: Universidad Nacional de la Patagonia San Juan Bosco, (2): 55-60.

Universidad Nacional Autónoma de Nicaragua, Managua. (2014). Programa de Asignatura de Prácticas de Especialización de la carrera Ciencias Sociales. UNAN-Managua.

Universidad Nacional Autónoma de Nicaragua, Managua. (2016). Plan de Estudios de la Carrera Ciencias Sociales, Facultad de Educación e Idiomas. UNANManagua.

Urra, U., Sandoval, S. y Irribarren, F. (2017). El desafío y futuro de la simulación como estrategia de enseñanza en enfermería. Investigación en Educación Médica, 6(22):119-125. 